\title{
Numerical study of dynamic noseleaf models in greater horseshoe bats, Rhinolophus ferrumequinum
}

\author{
Li Gao ${ }^{1}$, Hongwang $\mathrm{Lu}^{3}$, Jianxiong Feng ${ }^{4}$, Zhiwei Zhang ${ }^{5}$, Xin $\mathrm{Ma}^{3}$, and Weikai $\mathrm{He}^{1,2, *}$ \\ ${ }^{1}$ School of Physics and Technology, University of Jinan, No. 336, West Road of Nan Xinzhuang, 250022 Jinan, China \\ ${ }^{2}$ University of Jinan, Shandong Provincial Key Laboratory of Network based Intelligent Computing, 250022, Jinan, China \\ ${ }^{3}$ School of Physics, Shandong University, Jinan 250022, Shandong, China \\ ${ }^{4}$ School of Mechanical Engineering, Purdue University, West Lafayette, IN 47907, USA \\ ${ }^{5}$ School of Radiology, Taishan Medical University, Taian 271016, Shandong, China
}

\begin{abstract}
Echolocating greater horseshoe bats (Rhinolophus ferrumequinum) emit biosonar pulses through nostril. The nostrilis surrounded by sophisticated and delicate appendages, i.e. noseleaf. It is known that the static part of noseleaf can cause different effects on bat biosonar pulse. In addition, the dynmaic noseleaf was found to be able to shape their emission beam. For detailed investigation of the effects of dynamic lancet on the ultrasonic beam, a 3D noseleaf model was constructed using the micro CT scanning of the noseleaf samples, and a simple model was constructed digitally for comparison. The model consists of two parts, for which one is the triangle on the top and the other one is the rectangle at the bottom. Quantitative numerical investigation on the lancet dynamic effect on the ultrasonic beam was performed using finite element analysis for both models.
\end{abstract}

\section{Introduction}

Different species of bats have developed different echolocation strategy to adapt to their living environment. For example, horseshoe bats need to utilize active, negative and random sonar for precise echolocation when preying $[1,2]$. This poses great challenge to the echolocation system of bats. To satisfy the special needs of detection, horseshoe bats have developed some unique features. Foremost, horseshoe bat emits sonar through nostril rather than mouth, and it has developed complex noseleaf that can reflect the acoustic wave [3]. Instead of being as simple as manmade sound-emission baffles, such as loudspeaker, the noseleaf of greater horseshoe bat is sophisticated and comprise of three parts: lancet, sella and anterior leaf [4], which play different roles in shaping the beampattern. The coorperative motion of those structures can manipulate the far-field beampattern, thus widening the field view to avoid losing the target, so as to counteract evasive maneuvers of the prey. In the echo-locating process, the pinna and noseleaf of bats can exert critical effect on the acoustic field both in static status and in motion. The outer ears (pinna) of horseshoe bats deform during the echolocation process, thus improving the encoding ability of bats [5]. The more deformation the pinna has, the higher total energy the sidelobes possess. Except for the outer ear, the anterior part of noseleaves can also undergo non-rigid deformation. The deformation of anterior leaf is closely related to the biosonar pulse [6].
It is also known that the lancet plays an important part in determining the sonar pulse beamwidth which acts as resonance cavities that widen the biosonar beam in elevation at low frequencies of the biosonar pulse [7, 8]. Part of the time-varying characteristics in the bats echo-location system presents a novel way of information encoding, which provides inspiration for the development of new time-varying sonar sensor. In addition, the bats echo-location system characteristics can also be applied to the smart antenna design to enhance the performances of the acoustic devices, such as radar etc. [9-11].

Lancet can manipulate the beamwidth and the beampattern shapes in sonar pulses [4]. In horseshoe bats, it has been observed by the author and other researchers that while emitting biosonar pulses, the lancet of the noseleaf is conspicuously in non-rigid motion, in addition, the lancet motion shows time overlap with the biosonar pulse [4]. However, the focus of the previous work was the experimental results analysis, while the simulation results were mainly on the qualitative description of the effect of lancet on beampattern. Based on the previous study, the present work focuses more on the detailed investigation of the numerical acoustic effects of the lancet deformation. The goals of this research were to: 1) investigate the detailed influence that deformation of the lancet may have on the emitted biosonar pulses in the dynamic biosonar system using numerical method, thus providing fundamental data from biological properties for real mechanical application; 2) investigate the acoustical beampattern of a simple model that simulates the shape of the nose leaf, thus deepening

Corresponding author: sps_heweikai@yahoo.com 
the understanding of the interaction between the noseleaf structure and outgoing acoustic signals. The results calculated from one simplified geometry and one scanned model is compared.

\section{Models and methods}

One bat noseleaf sample was scanned using a micro-CT machine (Skyscan 1172; Bruker micro-CT, Konitch, Belgium), to construct a 3D digital surface mesh model (Fig.1 (a)). The model is the same model as male 2 in [4]. As shown in Fig.1(a), the part being circled with red dot represents the sound source. A simplified noseleaf geometry was constructed digitally, as shown in Fig.1(b). The model consists of two parts, for which one is the triangle on the top and the other one is the rectangle at the bottom. The triangle part represents the lancet. The two circles are two point sound sources, which represents a bipole array. The model ignores the sella structure and furrows on the lancet for more efficient modelling and lower computational cost. The effects of this simplification are discussed later. The individual 3D digital noseleaf models were handled using the software 3DMax (Autodesk, Mill Valley, CA, USA), to provide a good fit to the subsequent frame positions of the bend lancet from video recordings of lancet rotation. The original angle between lancet and reference plane, which is vertical to the ground base, is $\alpha$. In order to make the simulation results comparable, the initial angle between lancet and reference for the real model and simple model is the same, which is $48^{\circ}$ in this study. In addition, the angle $\alpha^{\prime}$ is the lancet rotation angle, which is relative to the initial position of the lancet. In this study, for both models, the angle $\alpha^{\prime}$ ranges from $0^{\circ}$ to $12^{\circ}$ with step size as $4^{\circ}$.

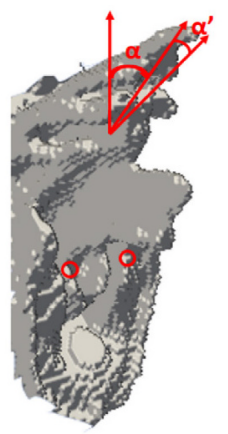

(a)

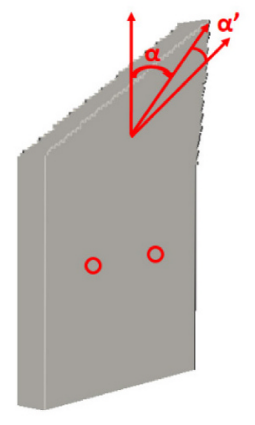

(b)
Fig. 1. Model of the bat noseleaf. (a) Scanned real noseleaf model; (b) simplified noseleaf model.

After obtaining the geometrical models, the both two 3D mesh models of noseleaf were employed to predict the acoustic field numerically using finite element method (FEM), investigating the effect of lancet by comparing the difference of biosonar pulse in different lancet deformation status. The numerical scheme used in this study consists of a combination of finite element simulation of the near-field acoustic pressure and the forward projection representing the far-field acoustic pressure [7, 12]. The FEM scheme is developed based on the Helmholtz equation,

$$
\Delta \phi+k^{2} \phi=-\mathrm{b}
$$

where $\mathrm{k}$ is the wave number, $\phi$ is the sound pressure, $\mathrm{b}$ is the source force term. In the finite element expression, the element stiffness matrix can be derived by the equation,

$$
K_{i j}=\Delta K_{i j}-k^{2} \Delta M_{i j}=\int_{\mathrm{V}} \nabla N_{i}^{T} \nabla N_{j}-k^{2} N_{i} N_{j} d V
$$

where $K_{i j}$ is the stiffness term, $M_{i j}$ is the mass term, $N_{i}$ is the $i_{\text {th }}$ shape function, $V$ is the volume of the element. On the boundary of the finite element domain, the stiffness matrix is given by,

$$
K_{i j}=\Delta K_{i j}-k^{2} \Delta M_{i j}+\mathrm{j} k \Delta C_{i j}
$$

where $\mathrm{Cij}$ is the damping term.

In the far-field, the projection is performed using Kirchhoff integral [5],

$$
\Phi(\boldsymbol{x})=-\frac{1}{4 \pi} \int_{S} \frac{e^{i k R}}{R} \boldsymbol{n}\left[\nabla \Phi+j k\left(1+\frac{j}{k R}\right) \frac{\boldsymbol{R}}{R} \Phi\right] d s
$$

where $\mathbf{R}$ is the vector between the surface element and the calculated position $\mathbf{x}, \mathbf{n}$ is the outward-pointing vector normal to the surface. Using the aforementioned equations, the acoustic field at both near field and far field can be derived. In this study, the far-field beampattern is of our interest.

After the far-field beampattern is obtained, the influence of lancet deformation on beampattern can be analysed by analysing the energy concentrated within the $-3 \mathrm{~dB}$ level contour line. In this study, 1) the energy distribution between the south and north globe within $3 \mathrm{~dB}$ contour line and 2) the overlap rate of lobes within $3 \mathrm{~dB}$ level line for the beampattern between initial and different deformation status of the lancet, are discussed. The overlapped area is added only once when calculating the area of $-3 \mathrm{~dB}$ lobes [12]. The overlap rate is a criterion for the degree of main lobe change, and those two parameters show negative correlation.

\section{Results}

The beampatterns corresponding to the respective noseleaf geometries are calculated in the frequency range from 60 to $80 \mathrm{kHz}$ with a $5 \mathrm{kHz}$ interval (Fig.2). For the real noseleaf model, the beampatterns are shown in Fig.2(a). The numerical results were displayed from another angle of view in reference [4]. However, neither the detailed explanation of the beampatterns was provided in the previous study, nor the beampatterns were compared to a manufactured model. As shown in Fig.2(a), in the initial status, there is an evident mainlobe, while there is no obvious sidelobe (not include in the $3 \mathrm{~dB}$ contour line). After $4^{\circ}$ of lancet rotation, the mainlobe became wider in the direction of elevation. For the low frequency range, some sidelobes extruded from the mainlobe, but they were still attached to it. When the lancet rotation reached $8^{\circ}$, one lobe separates from the mainlobe at low frequency, and the lobe attached itself to the mainlobe again with the increase of frequency. When the lancet rotation reached $12^{\circ}$, the beampattern is like 
$8^{\circ}$ rotation case. However, the sidelobes exhibited more significant separation in this case. It can be seen that the mainlobe width in elevation increases with incrementing lancet flexion. As shown in Fig.2(b), for the simple model, the energy is more dispersed compared to the real model, and there is no obvious mainlobe and sidelobe. As the lancet rotates, the change in the shape of the energy mainlobes of the simple model is not as large as the real model. In addition, the area with $-3 \mathrm{~dB}$ line of the beampatterns for this model is larger at high frequencies, which means the high frequency signal components has larger detection area, while for the results of the real noseleaf model, the trend is not obvious.

\section{$60 \mathrm{kHz} 65 \mathrm{kHz} 70 \mathrm{kHz} 75 \mathrm{kHz} 80 \mathrm{kHz}$}

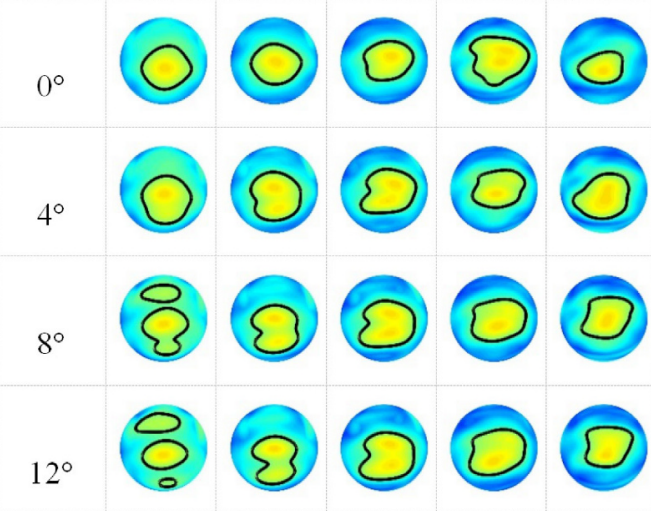

(a) Real noseleaf model

$60 \mathrm{kHz} 65 \mathrm{kHz} 70 \mathrm{kHz} 75 \mathrm{kHz} 80 \mathrm{kHz}$

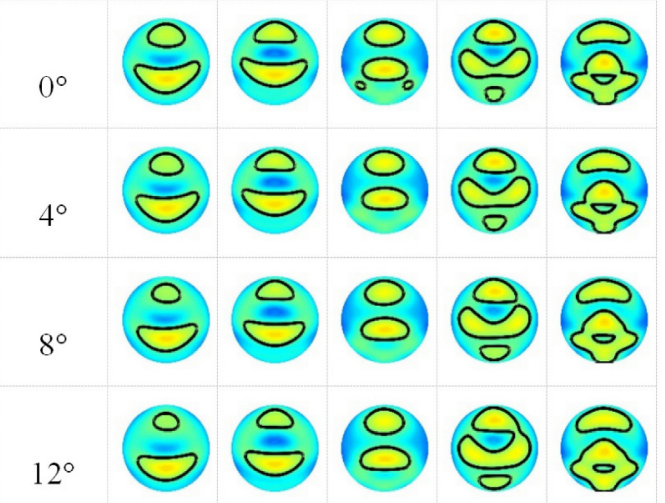

(b) Simple noseleaf model

Fig. 2. Numerical beampattern estimates obtained for the lancet rotation of noseleaf models. Each column represents beampattern under different lancet deformation, from posterior to anterior. Each row represents different beampattern in different frequencies.

For the simple model, the energy lobes show more location change rather than shape change (Fig.2 (b)). To obtain more straightforward description of the energy spatial distribution, the average energy ratio o between the south part and north part of the $-3 \mathrm{~dB}$ area in all the frequencies' beampatterns are calculated, corresponding to downward and upward direction on which bat focus attention. As shown in the Fig.3, for the real model, the energy moves to south when the deformation starts. However, the lancet rotates to $8^{\circ}$, the mainlobe energy returns to the north part. For the simple model, the energy moves to south continuously as the lancet rotates. The difference is hypothesized to be caused by the acoustic effect of the sella structure. As the lancet rotates, it will come closer to sella. The acoustic wave will interact between those two structures, thus causing significant influence on the acoustic directivity of the outgoing sound.

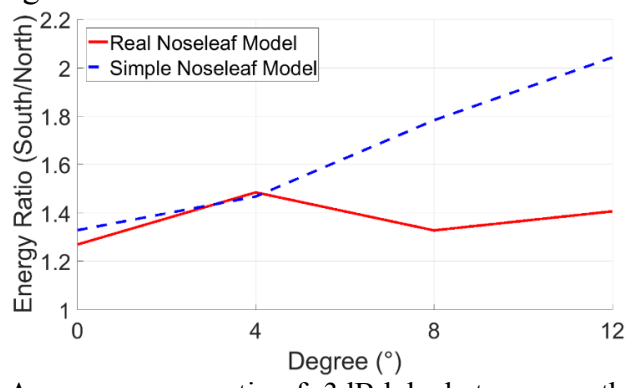

Fig. 3. Average energy ratio of $-3 \mathrm{~dB}$ lobe between south and north globe of the beampatterns.

The $-3 \mathrm{~dB}$ contour line in accord with different lancet flexion are compared in Fig. 4 for both models. For the real model, as shown in Fig.4(a), it can be found that: the change of $-3 \mathrm{~dB}$ lobes can be proportional change (Fig.4(a)g-i), directional change (Fig.4(a)m-o), shape change (Fig.4(a)d-f), or complicated combined directional and shape change (Fig.4(a) a-c). Compared to the read model, the $-3 \mathrm{~dB}$ lobes for the simple model does not show too much change in shape. The only obvious change is that the $-3 \mathrm{~dB}$ moves to the south when the lancet rotates.

The change of the overlap degree between the area within the $-3 \mathrm{~dB}$ contour can be quantitatively described by the beam overlap rate between initial lancet $(0$ rotation) and deformed lancet $(40,80,120$ rotation). As shown in Fig.5, for the simple model, the overlap becomes smaller with the increase of the lancet flexion, which is intuitive that the increase in the structural change will result in different directivity of the outgoing sound. However, for the real model, the overlap actually becomes smaller with larger structural deformation at high frequencies $(75,80 \mathrm{kHz})$, which is counterintuitive. On the other hand, it can be seen that the highest overlap rate occurs at $70 \mathrm{kHz}$ for the real model, while it occurs at $80 \mathrm{kHz}$ for the simple model. The quantitative results provide further support that, for the real noseleaf geometry, the sophistication of the structure exerts complicated effects on the radiated acoustic beampattern, which cannot be modeled by simple geometries.

To make the energy distribution easier to be compared for different frequencies, the $-3 \mathrm{~dB}$ contour for all the frequencies are plotted together in the same graph with different colours, as shown in Fig.6. For the real model, the $-3 \mathrm{~dB}$ lobe of sonar beams are concentrated at different frequencies without lancet deformation (Fig.6(a)). When the lancet flexion reached $12^{\circ}$, the shapes of the mainlobes change significantly and sidelobes becomes much larger (Fig.6(a')). For the simple model results, the lancet flexion also cause some difference in the energy distribution at different frequencies. 
$60 \mathrm{kHz} 65 \mathrm{kHz} 70 \mathrm{kHz} 75 \mathrm{kHz} 80 \mathrm{kHz}$

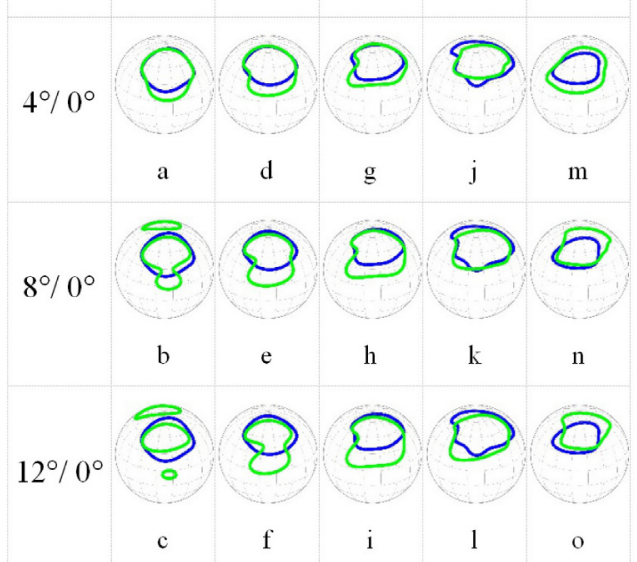

(a) Real noseleaf model

$60 \mathrm{kHz} 65 \mathrm{kHz} 70 \mathrm{kHz} 75 \mathrm{kHz} 80 \mathrm{kHz}$

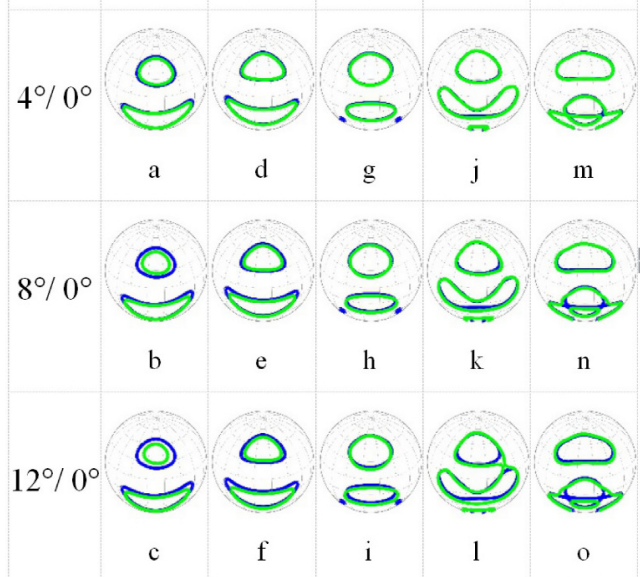

(b) Simple noseleaf model

Fig. 4. Beampattern overlap within $-3 \mathrm{~dB}$ level line of noseleaf model. Mainlobe without lancet deformation (blue lines) is compared with $4^{\circ}$ rotation, $8^{\circ}$ rotation, $12^{\circ}$ rotation (green lines).

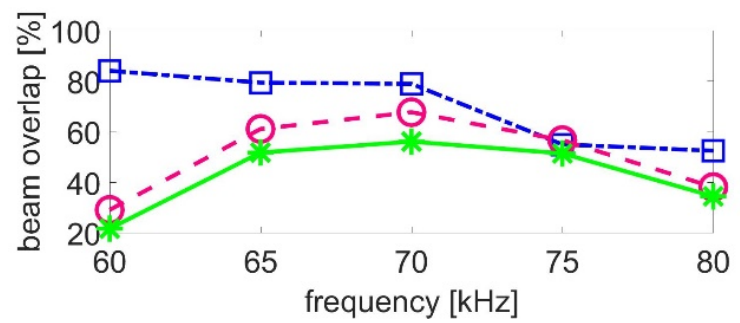

(a) Real noseleaf model

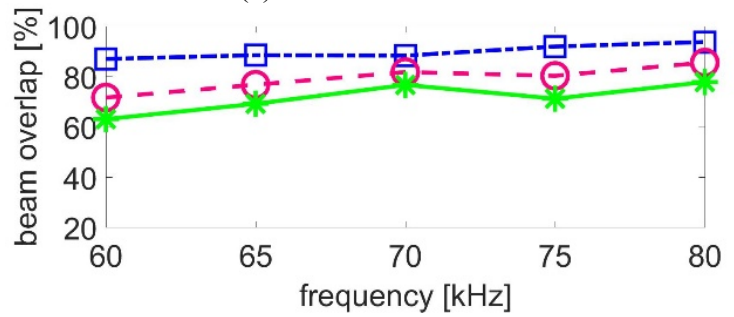

(b) Simple noseleaf model

Fig. 5. The overlap rate of beampattern within $-3 \mathrm{~dB}$ line between initial and different lancet flexion conditions. "व": overlap rate between $0^{\circ}$ and $4^{\circ}$ rotation of lancet (blue); "०": overlap rate between $0^{\circ}$ and $8^{\circ}$ rotation of lancet (red); “*”: overlap rate between $0^{\circ}$ and $12^{\circ}$ rotation of lancet (green).

\section{Discussions}

It is shown from the numerical results of the real noseleaf model that the biosonar pulse beampattern displays significant change with the motion of lancet (Fig.2(a)). When preying, the bat flight course is not fixed. In complicated environment, the biosonar beam needs to be adjusted spatially and directionally in the whole frequency range to satisfy the need of detection of prey. The motion of lancet, instead of turning around, which utilizes frequency modulated acoustic wave can perform the task (Fig.6(a')). The shape and direction of sonar pulse beam are very sensitive to the lancet deformation (Fig.6(a), (a')), which can achieve wider range of detection, thus improving the adaptability of bats to the environment. This shows that the enlargement of detection area of mainlobe and the sidelobes generation are significant features for greater horseshoe bats when lancet deforms. In the biology aspect, this feature can make up for one defection of CF-FM bats in rest, that is, there is only faint sidelobes besides mainlobe in the acoustic field during the pulse emission process for the noseleaf in static status. In addition, the phenomenon observed in this study supports the hypothesis that the potential function of these dynamic effects lies in enhancing the ability to encode useful sensory information, as the beampattern projects a weighted illumination on the environment and determines the contribution of each scatterer to the echo [5]. Nevertheless, the present results are not strong enough to determine the content of the useful sensory information to horseshoe bats. More investigation that can relate the effects of noseleaf on the biosonar in the bats hunting behavior can be done later.

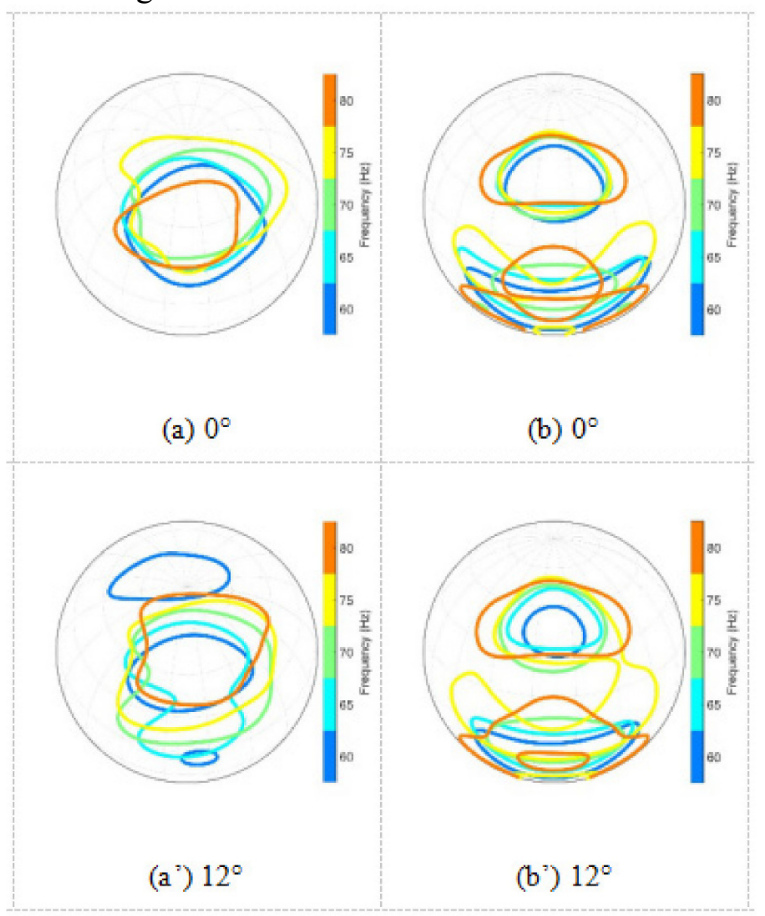

Fig. 6. Comparison the beampatterns of lancet from greater horseshoe bats in an upright (top row) and a bent pose (bottom row). (a) (a') Real noseleaf model; (b) (b') simple noseleaf model. 
While for the model with simple geometries, there is no obvious mainlobe or sidelobes, which means the acoustic energy is more dispersed in the predicted beampattern. In addition, the trend of the beampattern change with lancet deformation also has some discrepancies with the scanned noseleaf model results, especially for large deformation of the lancet. The difference in the FEM results is hypothesized to be caused by: (1) the absence of the sella structure in the simplified model; (2) the simplification of the noseleaf surface to flat surfaces without furrows and other substructures, which ignores the complicated noseleaf surface details. In the future study, more lancet flexion angles and more complicated synthesized structures should be investigated to reveal the relation between the noseleaf structure and emitted acoustics signals.

\section{Ethics Statement}

All animal work has been conducted in accordance with the relevant guidelines and regulations of the People's Republic of China. Permission for animal collections was granted by the managers of the land on which the caves were located and the animals were taken from their roosts. Greater horseshoe bats have been assessed as a species of "least concern" by the IUCN Red List of Threatened Species [13]. Experiments were carried out under institutional permit number 2012-377 from the Shandong University---Virginia Tech International Laboratory Animal Care Committee that specifically approved this study.

The first two authors, Li Gao and Hongwang Lu, contributed equally to this paper. Thanks to professor, Dr. Rolf Mueller for his advice and support in this study. This work was supported by the National Science Foundation of China under Grant [11704154, 11404140, 61271453]; Shandong Province under Grant [ZR2016AB13, BS2013SF001].

\section{References}

1. G. Jones, JMV, Rayner, Behavioral Ecology \& Sociobiology. 25, 183-191(1989)

2. G. Neuweiler, W. Metzner, U. Heilmann, R. Rübsamen, M. Eckrich, Behavioral Ecology \& Sociobiology, 20, 53-67(1987)

3. D. R. Griffin, Listening in the Dark. Cornell University Press, Ithaca (1986)

4. W. He, S. C. Petterson, A. K. Gupta, J. A. Simmons, R. Mueller, PLOS ONE. 10, e0121700 ( 2015)

5. L. Gao L, S. Balakrishnan, W. He, Z. Yan, R. Müller, Phys Rev Lett., 107, 214301 (2011)

6. L. Feng, L. Gao, H. Lu, R. Müller, PLOS ONE. 7, e346852012 (2012)

7. Q. Zhuang, R. Müller, Phys Rev E. 76, 051902-11 (2007)

8. Q. Zhuang, R. Mueller, Phys Rev Lett., 97, 2187014 (2006)

9. R. Müller, J. T. C. Hallam, 50, 131-145 (2005)
10. R. Müller, R. Kuc, Biomim. 2, S146-S161(2007)

11. JA. Flint, IEEE Antennas and Wireless Propagation Letters. 5, 145-147 (2006)

12. Z. Zhang, S. N. Truong, and R. Mueller, Phys Rev Lett. 103, 038701 (2009)

13. S. Aulagnier, A. M. Hutson, F. Spitzenberger, J. Juste, A. Karataş, J. Palmeirim \& M. Paunovic M, The IUCN Red List of Threatened Species. E. T19517A8947355 (2008) 